\title{
Farnesyltransferase inhibitors prevent HIV protease inhibitor (lopinavir/ritonavir)-induced lipodystrophy and metabolic syndrome in mice
}

\author{
TOMOKAZU TANAKA $^{1}$, HARUMASA NAKAZAWA ${ }^{1,2}$, NAOHIDE KURIYAMA ${ }^{1,2}$ and MASAO KANEKI ${ }^{1,2}$ \\ ${ }^{1}$ Department of Anesthesia, Critical Care and Pain Medicine, Massachusetts General Hospital, Harvard Medical School, \\ Charlestown, MA 02129; ${ }^{2}$ Department of Research, Shriners Hospitals for Children, Boston, MA 02114, USA
}

Received September 24, 2016; Accepted January 20, 2017

DOI: $10.3892 / e t m .2017 .5526$

\begin{abstract}
Highly active antiretroviral therapy (HAART) has successfully reduced the mortality rate of patients with human immune deficiency virus (HIV) and HIV protease inhibitors (HIV PIs) are key components of HAART. Complications of HAART, particularly those associated with HIV PIs including lipodystrophy and metabolic disturbance, have emerged as an important public health issue. No specific treatment is available to prevent and/or treat HIV PI-associated lipodystrophy and metabolic syndrome. The present study demonstrated that a relatively low-dose of farnesyltransferase inhibitor (FTI), tipifarnib ( $3 \mathrm{mg} / \mathrm{kg} / \mathrm{day}$, subcutaneous injection) and lonafarnib ( $5 \mathrm{mg} / \mathrm{kg} / \mathrm{day}$, subcutaneous injection), prevented the onset of lipodystrophy and metabolic syndrome induced by the combination of two HIV PIs, lopinavir $(50 \mathrm{mg} / \mathrm{kg} / \mathrm{day}$, intraperitoneal injection) and ritonavir $(12.5 \mathrm{mg} / \mathrm{kg} /$ day, intraperitoneal injection), in mice. Consistent with previous studies, treatment with lopinavir/ritonavir for 2 weeks decreased body weight, adipose tissue mass, levels of plasma adiponectin and leptin, and increased plasma levels of triglycerides, total cholesterol and insulin. Tipifarnib and lonafarnb prevented or ameliorated all of these alterations
\end{abstract}

Correspondence to: Dr Masao Kaneki, Department of Anesthesia, Critical Care and Pain Medicine, Massachusetts General Hospital, Harvard Medical School, 149 Thirteenth Street, Room 6604, Charlestown, MA 02129, USA

E-mail: mkaneki@helix.mgh.harvard.edu

Abbreviations: HAART, highly active antiretroviral therapy; HIV, human immune deficiency virus; PI, protease inhibitor; AIDS, acquired immune deficiency syndrome; ART, antiretroviral therapy; $\mathrm{CD}$, cluster of differentiation; HMG-CoA, hydroxymethylglutaryl-CoA; HGPS, Hutchinson-Gilford progeria syndrome; FTI, farnesyltransferase inhibitor; SC, subcutaneous; IP, intraperitoneal; FTase, farnesyltransferase; LPV, lopinavir; RTV, ritonavir; DMSO, dimethyl sulfoxide; ANOVA, analysis of variance

Key words: HIV protease inhibitor, lipodystrophy, metabolic syndrome, farnesyltransferase inhibitor, AIDS in the HIV PI-treated mice. These data identify FTIs as a novel potential strategy to prevent or treat HIV PI-associated lipodystrophy and metabolic syndrome in HIV-infected patients on HAART.

\section{Introduction}

Human immunodeficiency virus (HIV) protease inhibitors (HIV PIs) are key components of highly active antiretroviral therapy (HAART) to treat patients infected with HIV. HAART has been highly successful in controlling HIV replication and reducing the morbidity and mortality rates of patients with acquired immune deficiency syndrome (AIDS) (1). However, significant adverse side effects are associated with long-term use of HIV PIs. Patients treated with HAART frequently develop a metabolic syndrome associated with partial lipodystrophy, hyperlipidemia and insulin resistance (2-4). The metabolic complications of HAART potentially increase the risk of cardiovascular disease in HIV-infected patients (4-6). Among others, HIV PIs are considered to serve a pivotal role in the development of HAART-related metabolic complications. Therefore, HIV PI-associated lipodystrophy and metabolic syndrome has emerged as an important public health issue. The number of patients with HIV diagnosed with cardiovascular disease been increasing and the metabolic complications of HAART are considered to be contributing to this increase $(7,8)$.

In 2015, World Health Organization issued a new guideline on antiretroviral therapy (ART) against HIV. The new guideline recommends that ART be initiated as soon as HIV infection is detected regardless of the progression of AIDS or reduced cluster of differentiation (CD) 4-positive cell count (9). This recommendation is based on evidence that earlier use of ART results in improved clinical outcomes (9). It is anticipated that a greater number of individuals living with HIV may be treated with HAART for a longer period of time by implementing this novel guideline worldwide. However, no specific treatment is currently available for the prevention and/or reversal of HAART-related metabolic complications. For example statins, as inhibitors of hydroxymethylglutaryl-CoA (HMG-CoA) reductase, are effective at reducing high cholesterol levels and have been prescribed to 
patients with HIV receiving HAART $(10,11)$. However, there are also a number of weaknesses and drawbacks of statin use to treat HIV PI-related metabolic syndrome. Statins increase the incidence of type II diabetes (12) and drug-drug interactions exist between some statins and HIV PIs (13). Statins are not capable of effectively improving insulin sensitivity or ameliorating lipodystrophy. In addition, statins are not so effective at reducing high triglyceride levels. Novel effective strategies to prevent or treat HAART-related metabolic complications are thus required.

The molecular mechanism by which HAART and/or HIV PIs cause metabolic disturbance is not well understood. Among others, the inhibition of zinc metalloprotease ZMPSTE 24 by HIV PIs has been proposed to serve an important role in the development of HAART-associated lipodystrophy and metabolic syndrome. ZMPSTE 24 is the key enzyme in the maturation of prelamin A, which is encoded by the $L M N A$ gene and some mutations in the LMNA or ZMPSTE 24 gene cause lipodystrophy and premature aging syndrome such as Hutchinson-Gilford progeria syndrome (HGPS) $(14,15)$. Lipodystrophy is a major characteristic of HGPS (16).

During the maturation process of prelamin A, following the farnesylation of prelamin A, ZMPSTE 24 cleaves farnesylated prelamin A (17-19). Protein farnesylation is a covalent attachment of farnesyl pyrophosphate to cysteine thiols in the CAAX motif located in the carboxyl terminus of proteins (' $\mathrm{C}$ ' is cysteine, ' $\mathrm{A}$ ' is aliphatic amino acid, and ' $\mathrm{X}$ ' is any amino acid at the carboxyl terminus, but typically serine, methionine, glutamine or alanine.) $(20,21)$. Protein farnesylation is catalyzed by farnesyltransferase (FTase) and promotes membrane translocation of the protein. Following this cleavage by ZMPSTE 24, mature lamin A no longer contains farnesylated cysteine (17-19). A previous study demonstrated that treatment with HIV PI, lopinavir (LPV), atazanavir or tipranavir, blocks ZMPSTE 24 activity and thereby accumulates farnesylated prelamin A in cultured fibroblasts (22). Accumulation of farnesylated prelamin A due to a genetic mutation in t LMNA or ZMPSTE 24 is considered to be a major factor in the pathogenesis of HGPS (23). Based on these data, the safety and efficacy of an FTase inhibitor (FTI), lonafarnib, has been assessed in ongoing clinical trials in pediatric patients with HGPS and promising results have been reported (24).

Lipodystrophy is a common feature of HGPS and HAART-related complications, although there are substantial differences in the signs and symptoms of these two diseases. Together with previous results indicating that HIV PIs inhibit ZMPSTE 24 leading to the accumulation of farnesylation of prelamin A in cultured cells (22), these previous findings raise the possibility that FTIs may prevent the development of HIV PIs-induced lipodystrophy and metabolic syndrome in addition to HGPS. A previous study determined that FTI-277 ameliorates the adverse effects of HIV PIs, ritonavir and the combination of LPV and ritonavir (RTV), in cultured human coronary artery endothelial cells (25). However, the effects of FTIs have not yet been studied in vivo in an animal model of HIV PI- or HAART-induced metabolic disturbances. Therefore, the present study demonstrates the preventive effects of relatively low doses of tipifarnib and lonafarnib, clinically applicable FTIs, on lipodystrophy and metabolic syndrome induced by the combination of LPV and RTV in mice.

\section{Materials and methods}

Animal treatments. A total of 32 male C57BL/6 mice (22.0-25.4 g) at 8 weeks of age (Jackson Laboratory, Bar Harbor, ME, USA) were used in the present study. The mice were housed in a pathogen-free animal facility maintained at $25^{\circ} \mathrm{C}$, with relative humidity of $50 \pm 10 \%$, and illuminated by a $12-\mathrm{h}$ light-dark cycle. The mice were provided with standard rodent chow and water ad libitum. The mice received daily intraperitoneal injections of $50 \mathrm{mg} / \mathrm{kg} /$ day LPV (Cayman Chemical, Ann Arbor, MI, USA) and $12.5 \mathrm{mg} / \mathrm{kg} /$ day RTV (Selleck Chemicals, Houston, TX, USA) or vehicle (10\% ethanol/15\% propylene glycol in normal saline) alone for 2 weeks as previously described (26). Simultaneously, the mice were treated with daily subcutaneous injections of $3 \mathrm{mg} / \mathrm{kg} /$ day tipifarnib (R11577), $5 \mathrm{mg} / \mathrm{kg} /$ day lonafarnib (SCH66336) (both from Selleck Chemicals) or vehicle [5\% dimethyl sulfoxide (DMSO) in $0.1 \mathrm{ml}$ normal saline] alone for 2 weeks. Therefore, the following 4 treatment groups were used in the current study (n=8 mice/group): Group 1, vehicle for LPV/RTV + vehicle for FTIs; group 2, LPV/RTV + vehicle for FTIs; group 3, LPV/RTV + tipifarnib; and group 4, LPV/RTV + lonafarnib. A total of 4 mice of each group were reared in the same cage. Lopinavir and ritonavir were diluted in a vehicle of $10 \%$ ethanol/15\% propylene glycol in normal saline (26). Tipifarnib and lonafarnib were dissolved in DMSO at $10 \mathrm{mg} / \mathrm{ml}$ and then diluted in $0.1 \mathrm{ml}$ normal saline. Just before the inception of treatment (day 1) and 7 (day 8) and 14 days (day 15) thereafter, blood glucose and body weight were measured. Blood glucose levels were measured using a blood glucose tester (Bayer Corporation, Mishawaka, IN, USA). Food intake was assessed on day 8 and day 15 by calculating the weekly loss of the chow provided to the mice. On day 15 , following 4 -h fasting, mice were anesthetized with an intraperitoneal injection of pentobarbital sodium (50 mg/kg; Lundbeck US, Deerfield, IL, USA) and adipose tissues (inguinal, epididymal, and retroperitoneal white adipose tissues and interscapular brown adipose tissue) were excised following laparotomy. The mice were then euthanized by exsanguination under anesthesia with pentobarbital sodium, and blood samples were collected by cardiac puncture. All experiments were completed in accordance with the institutional guidelines and the study protocol was approved by the Institutional Animal Care and Use Committee (IACUC) at the Massachusetts General Hospital (protocol no. 2015N000216; Charlestown, MA, USA). The animal care facility is accredited by the Association for Assessment and Accreditation of Laboratory Animal Care.

Biochemical assays. Plasma samples were obtained by centrifugation of the heparinized blood at 2,200 $\mathrm{x}$ g for $20 \mathrm{~min}$ at $4^{\circ} \mathrm{C}$ and stored at $-80^{\circ} \mathrm{C}$ until the biochemical assays were performed. Levels of triglycerides, total cholesterol, free fatty acids (also known as nonesterified fatty acids) in plasma were measured colorimetrically using commercially available kits [a LabAssay ${ }^{\mathrm{TM}}$ Triglyceride (cat no. 290-63701) for triglycerides, a cholesterol assay kit (cat no. 999-02601) for total cholesterol and a NEFA C kit (cat no. 279-75401) for free 
A

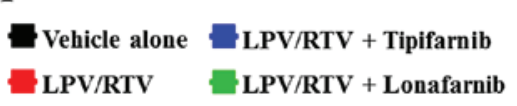

B
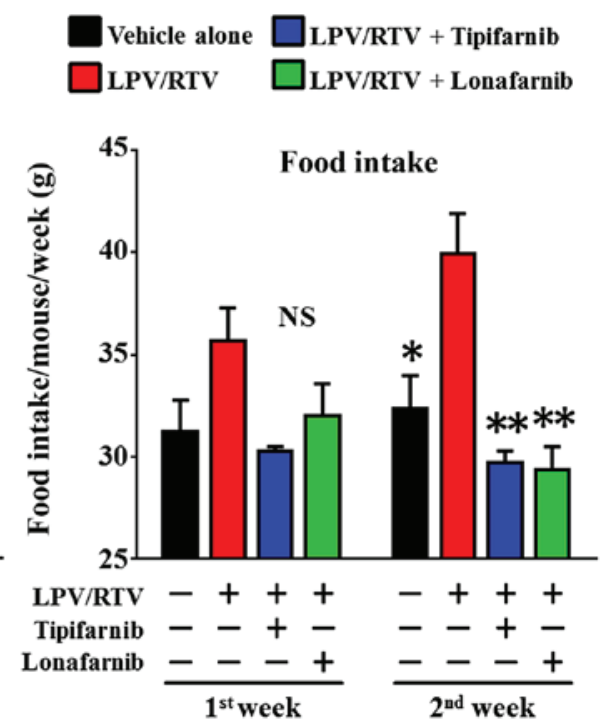

Figure 1. Effects of FTIs on body weight and food intake in mice treated with LPV/RTV. (A) Treatment with LPV/RTV for 2 weeks significantly decreased body weight compared with vehicle alone. The effect of LPV/RTV on body weight was attenuated by FTIs: When the mice were co-treated with tipifarnib or lonafarnib, LPV/RTV failed to significantly decrease body weight. (B) LPV/RTV significantly increased food intake in the second week of the treatment compared with vehicle alone. FTIs prevented LPV/RTV-induced increased food intake. In the first week of the treatments, no significant difference in food intake between the groups was observed, although LPV/RTV tended to increase it. ${ }^{*} \mathrm{P}<0.05,{ }^{* *} \mathrm{P}<0.01 \mathrm{vs}$. LPV/RTV treatment, $\mathrm{n}=8 \mathrm{mice}$ per group. FTI, farnesyltransferase inhibitor; LPV, lopinavir; RTV, ritonavir; NS, not significant.

fatty acids; Wako Chemicals USA, Inc., Richmond, VA, USA). Insulin, adiponectin and leptin concentrations in plasma were evaluated by ELISA according to the manufacturer's assay protocols of commercially available kits [an Ultra Sensitive Mouse Insulin ELISA kit (cat no. 90080) for insulin, a Mouse Adiponectin ELISA kit (cat no. 80569) for adiponectin and a Mouse Leptin ELISA kit (cat no. 90030) for leptin (Crystal Chem, Inc., Downers Grove, IL, USA)].

Statistical analysis. The data were analyzed by an analysis of variance assay (ANOVA) using GraphPad Prism 6.0 software (GraphPad Software, Inc., La Jolla, CA, USA). For comparison among the four groups, the data were compared with one-way ANOVA followed by Newman-Keuls multiple comparison test. $\mathrm{P}<0.05$ was considered to indicate a statistically significant difference. All data are expressed as means \pm standard error of the mean.

\section{Results}

FTIs prevented LPVIRTV-induced decreases in body weight and adipose tissue mass in mice. Treatment with LPV/RTV, commonly used HIV PIs, for 2 weeks significantly decreased the body weight and mass of adipose tissues in mice including epididymal, inguinal and retroperitoneal white adipose tissues and interscapular brown adipose tissue compared with mice treated with vehicle alone $(\mathrm{P}<0.05$; Fig. $1 \mathrm{~A})$. On day 7 following the inception of LPV/RTV treatment there was a trend toward decreased body weight in groups treated with LPV/RTV but no statistically significant difference was observed between the four groups. These results are in agreement with those of previous studies $(26,27)$. Notably, the body weight loss induced by LPV/RTV was not associated with reduced food intake; food intake was significantly increased in the LPV/RTV group compared with the mice treated with vehicle alone $(\mathrm{P}<0.05$; Fig. $1 \mathrm{~B})$ following two weeks treatment.

Tipifarnib and lonafarnib prevented or ameliorated the LPV/RTV-induced decreases in body weight and fat mass $(\mathrm{P}<0.05$; Fig. 2). Furthermore, the FTIs reversed the increased food intake in LPV/RTV-treated mice two weeks after treatment $(\mathrm{P}<0.01$; Fig. 1B). There was no significant difference in food intake between the mice receiving the combination treatment with LPV/RTV and tipifarnib or LPV/RTV and lonafarnib and those receiving vehicle alone (Fig. 1B).

FTIs prevented LPV/RTV-induced hyperlipidemia in mice. HIV PI-related lipodystrophy in patients infected with HIV is associated with hyperlipidemia $(28,29)$. Similarly, LPV/RTV increased plasma levels of triglycerides, total cholesterol and free fatty acids in mice (Fig. 3), consistent with results of previous studies in mice $(26,27)$. Tipifarnib and lonafarnib significantly prevented LPV/RTV-induced hypertriglyceridemia $(\mathrm{P}<0.05$; Fig. 3A) and hypercholesterolemia $(\mathrm{P}<0.001$; Fig. 3B). When the mice were co-treated with tipifarnib or lonafarnib, LPV/RTV failed to significantly increase circulating free fatty acids compared with mice treated with vehicle alone. There were trends toward decreased levels of free fatty acids by tipifarnib and lonafarnib in LPV/RTV-treated mice, but no statistically significant differences were observed (Fig. 3C).

FTIs reversed the effects of LPV/RTV on plasma insulin, adiponectin and leptin concentrations. Neither LPV/RTV nor FTIs altered blood glucose levels (Fig. 4A). By contrast, LPV/RTV significantly increased plasma insulin concentrations compared with vehicle alone $(\mathrm{P}<0.001$; Fig. 4B). 

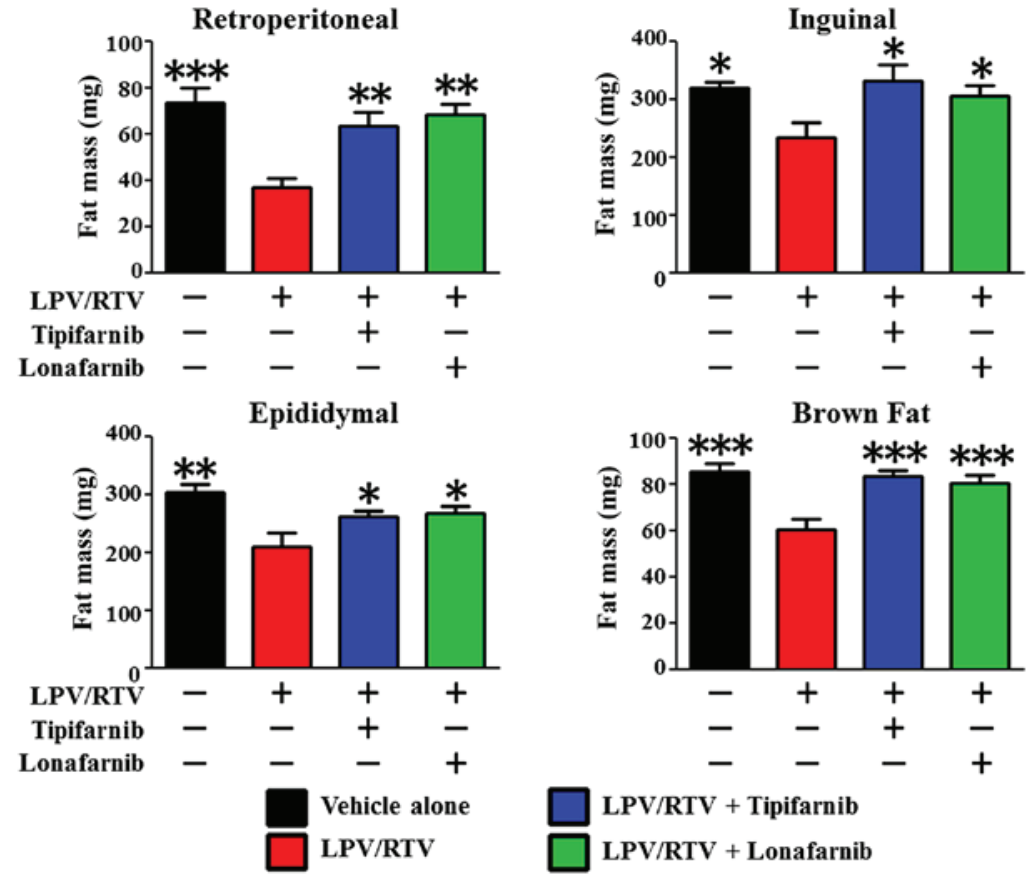

Figure 2. Effects of FTIs on LPV/RTV-induced reduction in adipose tissue mass in mice. LPV/RTV significantly decreased the mass of adipose tissues including retroperitoneal, inguinal, and epididymal white adipose tissues and interscapular brown adipose tissue compared with vehicle alone. FTIs almost completely prevented the LPV/RTV-induced decrease in fat mass in all adipose tissues. ${ }^{*} \mathrm{P}<0.05,{ }^{* *} \mathrm{P}<0.01,{ }^{* * * *} \mathrm{P}<0.001$ vs. LPV/RTV treatment, $\mathrm{n}=8$ mice per group. FTI, farnesyltransferase inhibitor; LPV, lopinavir; RTV, ritonavir.

A

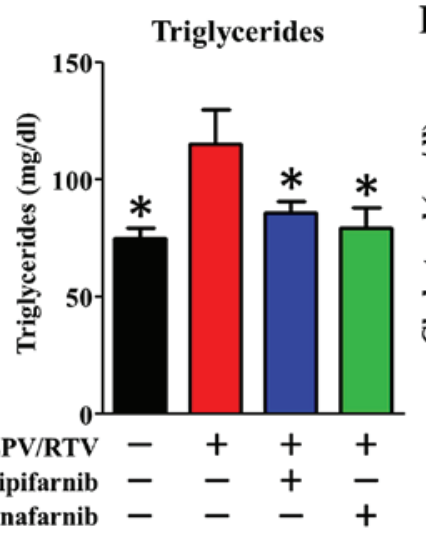

B

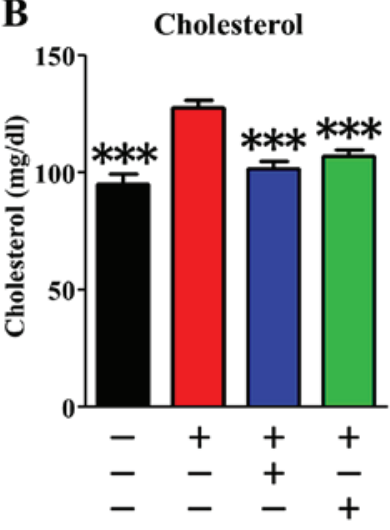

C Free fatty acids

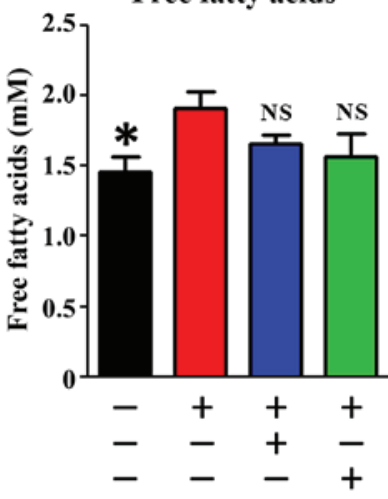

Figure 3. Effects of FTIs on LPV/RTV-induced hyperlipidemia in mice. LPV/RTV significantly increased plasma levels of (A) triglycerides, (B) total cholesterol and (C) free fatty acids compared with vehicle alone. When co-treated with tipifarnib or lonafarnib, LPV/RTV failed to significantly increase levels triglycerides, total cholesterol, or free fatty acids compared with vehicle alone. Tipifarnib and lonafarnib significantly decreased triglyceride and total cholesterol levels in LPV/RTV-treated mice to the levels observed in mice treated with vehicle alone. There were trends toward decreased levels of free fatty acids by the FTIs in mice treated LPV/RTV, but no statistically significant differences were observed between the mice treated with LPV/RTV alone and those co-treated with LPV/RTV and tipifarnib or LPV/RTV and lonafarnib. ${ }^{*} \mathrm{P}<0.05,{ }^{* * *} \mathrm{P}<0.001$ vs. LPV/RTV treatment, $\mathrm{n}=8$ mice per group. FTI, farnesyltransferase inhibitor; LPV, lopinavir; RTV, ritonavir; NS, not significant.

Tipifarnib and lonafarnib prevented hyperinsulinemia in LPV/RTV-treated mice $(\mathrm{P}<0.05$; Fig. 4B). These results indicate that LPV/RTV induced insulin resistance in mice, which was reversed by the FTIs.

Consistent with the LPV/RTV-induced insulin resistance and its reversal by the FTIs, LPV/RTV significantly decreased plasma level of adiponectin $(\mathrm{P}<0.001)$, an insulin-sensitizing adipokine, and the FTIs restored plasma adiponectin concentrations to the levels observed in the mice receiving vehicle alone (Fig. 4C). Similarly, LPV/RTV decreased plasma leptin levels $(\mathrm{P}<0.05)$ and this decrease was reversed by tipifarnib and lonafarnib (Fig. 4D).

\section{Discussion}

The present study demonstrates that a relatively low-dose of tipifarnib and lonafarnib prevents LPV/RTV-induced reductions in body weight and adipose tissue mass, hyperlipidemia 

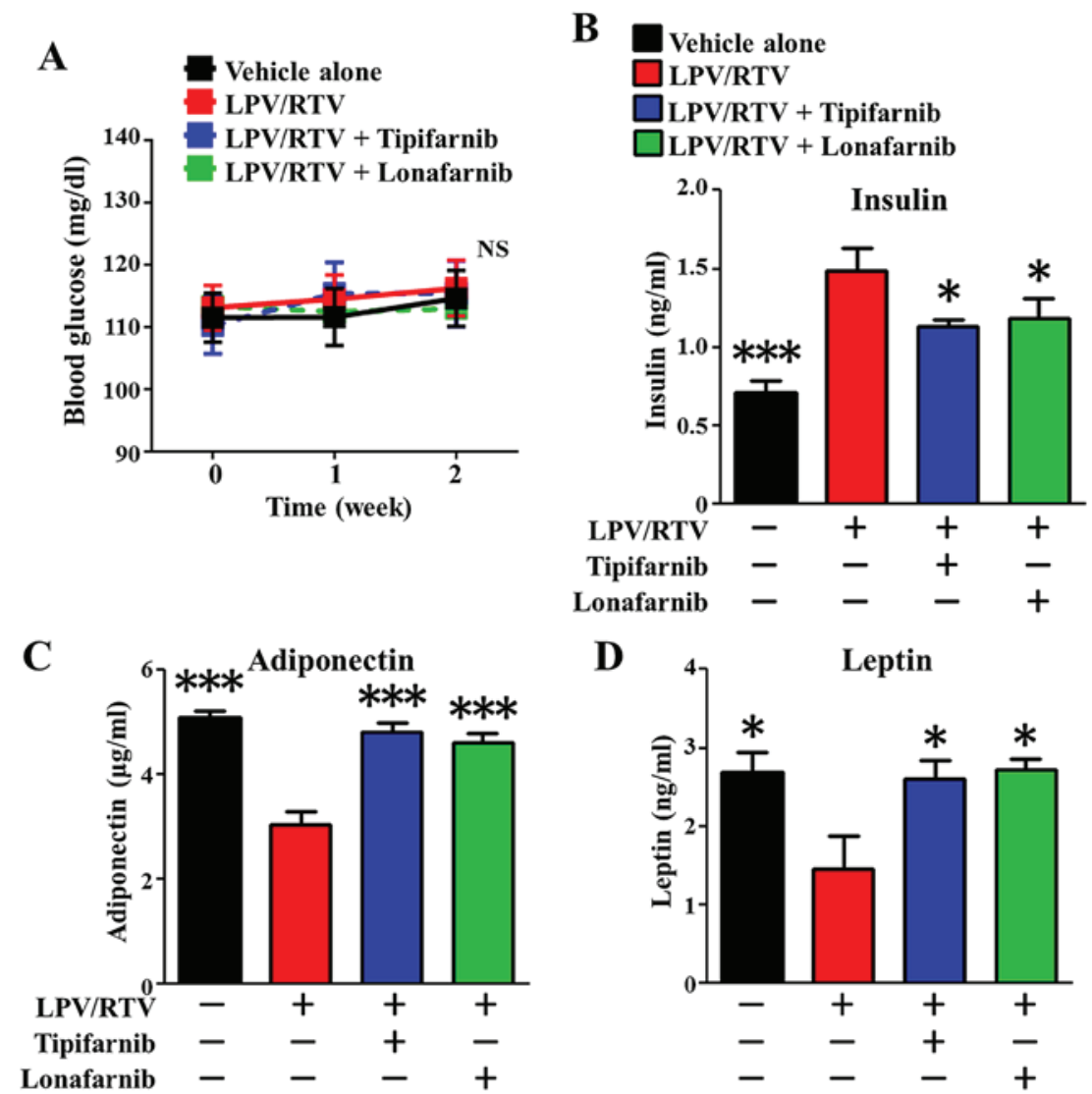

Figure 4. Effects of FTIs on plasma insulin, adiponectin and leptin levels in mice treated with LPV/RTV. (A) No differences were observed in blood glucose levels between the groups. (B) LPV/RTV significantly increased plasma insulin concentrations compared with vehicle alone. Tipifarnib and lonafarnib significantly inhibited the LPV/RTV-induced increase in plasma insulin concentrations. Plasma insulin concentrations in the mice receiving the combination treatment of LPV/RTV and tipifarnib or LPV/RTV and lonafarnib did not significantly differ from those treated with vehicle alone, although plasma insulin concentrations appeared to be greater in the mice treated with LPV/RTV and FTI compared with vehicle alone. Treatment with LPV/RTV significantly decreased plasma (C) adiponectin and (D) leptin concentrations compared with vehicle alone. Tipifarnib and lonafarnib reversed the effects of LPV/RTV on adiponectin and leptin concentrations. ${ }^{*} \mathrm{P}<0.05,{ }^{* * *} \mathrm{P}<0.001$ vs. LPV/RTV treatment, $\mathrm{n}=8$ mice per group. FTI, farnesyltransferase inhibitor; LPV, lopinavir; RTV, ritonavir; NS, not significant.

and hyperinsulinemia in mice. To the best of our knowledge, this is the first study focusing on the effects of FTI on HIV PI- or HAART-related complications in vivo. The results of the current study suggest that FTIs may be capable of preventing or ameliorating HIV PI-associated lipodystrophy and metabolic syndrome, including hyperlipidemia and insulin resistance, in patients with HIV undergoing treatment with HAART. It is indicated that protein farnesylation serves a role in the pathogenesis of HIV PI-induced metabolic disturbances in mice.

Treatment with LPV/RTV decreased body weight in mice. It should be noted, however, that LPV/RTV significantly increased food intake. Therefore, the decreased body weight observed in the LPV/RTV-treated mice may not be accounted for by toxic effects of HIV PIs alone, and that it may be attributable to the metabolic changes induced by LPV/RTV. Increased food intake was associated with reduced plasma leptin levels in LPV/RTV-treated mice. Leptin is an adipokine that is secreted by adipocytes and inhibits appetite and food intake (30). Previous studies have demonstrated that the level of circulating leptin is associated with fat mass in mice and humans $(30,31)$. It is therefore conceivable that fat mass loss may lead to decreased leptin production and secretion, which in turn, increases food intake in LPV/RTV-treated mice.
Adiponectin is a major adipokine that promotes insulin sensitivity (32). The level of circulating adiponectin is closely associated with insulin sensitivity. It is reasonable to speculate, therefore, that decreased adiponectin levels may contribute to the onset of insulin resistance, as indicated by hyperinsulinemia, in LPV/RTV-treated mice. Notably, previous studies have indicated that circulating levels of leptin and adiponectin are significantly decreased in patients with HAART-related lipodystrophy and metabolic syndrome (33-35).

LPV/RTV was selected in the present study to induce lipodystrophy and metabolic syndrome in mice based on the following reasons: The combination of LPV and RTV is a frequently used component of HAART for the treatment of HIV infection in adults, adolescents and children (36). In most, but not all countries, LTV/RTV is one of the preferred HIV PI treatments in the recommended second-line regimens (37). LPV/RTV treatment is associated with lipodystrophy and metabolic syndrome in HIV-infected patients $(38,39)$. Finally, to the best of our knowledge, the combination of LPV/RTV is the only HIV PI regimen that has been used to induce lipodystrophy and metabolic syndrome in rodents $(26,27)$.

Clinically relevant doses of LPV (50 mg/kg/day) and RTV $(12.5 \mathrm{mg} / \mathrm{kg} /$ day) in mice were used in the current study (26). 
The regular doses for daily oral treatment with LPV/RTV as a monotherapy or in combination with other ART in adult HIV patients are LPV (400-800 mg/day or $10 \mathrm{mg} / \mathrm{kg} /$ day) and RTV (100-200 mg/day or $2.5 \mathrm{mg} / \mathrm{kg} /$ day) (40).

The doses of the FTIs used in the current study, tipifarnib (3 $\mathrm{mg} / \mathrm{kg} /$ day, subcutaneous injection) and lonafarnib (5 mg/kg/day, subcutaneous injection), appear much lower compared with those used in a mouse model of HGPS (tipifarnib 150 or $450 \mathrm{mg} / \mathrm{kg} /$ day, oral administration) (41). However, in clinical trials of lonafarnib in pediatric patients with HGPS (70-150 mg/m² body surface area, twice daily, oral administration; Clinical Trials.gov identifiers: NCT00425607, NCT00879034, and NCT00916747), the differences in route of administration (subcutaneous injection vs. oral administration) and species (human vs. mouse) make precise comparisons difficult. Notably, previous clinical trials investigating patients with cancer and leukemia demonstrated the safety and tolerability of oral administration of tipifarnib (600 mg/day) and lonafarnib $(250 \mathrm{mg} /$ day $)(42,43)$. Previous studies reported that the bioavailability of oral tipifarnib administration was $\sim 27,30$ or $34 \%$ in healthy volunteers and/or patients with cancer (44-46). It is possible that higher doses of FTIs may be required to exert the protective effects in HGPS compared with LPV/RTV-induced lipodystrophy and metabolic syndrome, as HGPS is a fatal disease and characterized by more serious symptoms compared with the complications of HIV PIs. However, the possibility that the molecular mechanisms underlying the beneficial effects of FTIs may differ between HGPS and HIV PI-related metabolic disturbances cannot be excluded. Further studies are required to clarify whether this is the case.

In conclusion, the present study demonstrates that a relatively low-dose of tipifarnib and lonafarnib prevented LPV/RTV-induced lipodystrophy and metabolic syndrome in mice. These findings warrant a clinical trial to study the safety and efficacy of low-dose FTI on HAART-related metabolic complications in individuals living with HIV.

\section{Acknowledgements}

The present study was supported by the National Institutes of Health, Rockville, USA (grant no. R01GM115552) and the Institutional Fund of Massachusetts General Hospital, Charlestown, USA (grant no. 1200-212238).

\section{References}

1. Quinn TC: HIV epidemiology and the effects of antiviral therapy on long-term consequences. AIDS 22 (Suppl 3): S7-S12, 2008.

2. Yanovski JA, Miller KD, Kino T, Friedman TC, Chrousos GP, Tsigos $C$ and Falloon J: Endocrine and metabolic evaluation of human immunodeficiency virus-infected patients with evidence of protease inhibitor-associated lipodystrophy. J Clin Endocrinol Metab 84: 1925-1931, 1999.

3. de Waal R, Cohen K and Maartens G: Systematic review of antiretroviral-associated lipodystrophy: Lipoatrophy, but not central fat gain, is an antiretroviral adverse drug reaction. PLoS One 8: e63623, 2013.

4. Mondy K and Tebas P: Cardiovascular risks of antiretroviral therapies. Annu Rev Med 58: 141-155, 2007.

5. Boccara F: Cardiovascular complications and atherosclerotic manifestations in the HIV-infected population: Type, incidence and associated risk factors. AIDS 22 (Suppl 3): S19-S26, 2008.
6. Gleason RL Jr, Caulk AW, Seifu D, Parker I, Vidakovic B, Getenet H, Assefa G and Amogne W: Current Efavirenz (EFV) or ritonavir-boosted lopinavir (LPV/r) use correlates with elevate markers of atherosclerosis in HIV-infected subjects in Addis Ababa, Ethiopia. PLoS One 10: e0117125, 2015.

7. Friis-Møller N, Weber R, Reiss P, Thiébaut R, Kirk O, d'Arminio Monforte A, Pradier C, Morfeldt L, Mateu S, Law M, et al: Cardiovascular disease risk factors in HIV patients-association with antiretroviral therapy. Results from the DAD study. AIDS 17: 1179-1193, 2003.

8. Calza L, Manfredi R, Pocaterra D and Chiodo F: Risk of premature atherosclerosis and ischemic heart disease associated with HIV infection and antiretroviral therapy. J Infect 57: 16-32, 2008.

9. WHO Guidelines Approved by the Guidelines Review Committee: Guideline on when to start antiretroviral therapy and on pre-exposure prophylaxis for HIV. World Health Organization, 2015.

10. Dubé MP, Stein JH, Aberg JA, Fichtenbaum CJ, Gerber JG, Tashima KT, Henry WK, Currier JS, Sprecher D, Glesby MJ, et al: Guidelines for the evaluation and management of dyslipidemia in human immunodeficiency virus (HIV)-infected adults receiving antiretroviral therapy: Recommendations of the HIV medical association of the infectious disease society of America and the adult AIDS clinical trials group. Clin Infect Dis 37: 613-627, 2003.

11. National Cholesterol Education Program (NCEP) Expert Panel on Detection, Evaluation, and Treatment of High Blood Cholesterol in Adults (Adult Treatment Panel III): Third report of the national cholesterol education program (NCEP) Expert Panel on Detection, Evaluation, and treatment of high blood cholesterol in adults (Adult Treatment Panel III) final report. Circulation 106: 3143-3421, 2002.

12. Lichtenstein KA, Hart RL, Wood KC, Bozzette S, Buchacz K and Brooks JT; HIV Outpatient Study Investigators: Statin use is associated with incident diabetes mellitus among patients in the HIV outpatient study. J Acquir Immune Defic Syndr 69: 306-311, 2015.

13. Chauvin B, Drouot S, Barrail-Tran A and Taburet AM: Drug-drug interactions between HMG-CoA reductase inhibitors (statins) and antiviral protease inhibitors. Clin Pharmacokinet 52: 815-831, 2013.

14. Eriksson M, Brown WT, Gordon LB, Glynn MW, Singer J, Scott L, Erdos MR, Robbins CM, Moses TY, Berglund P, et al: Recurrent de novo point mutations in lamin A cause Hutchinson-Gilford progeria syndrome. Nature 423: 293-298, 2003.

15. Caron M, Auclair M, Donadille B, Béréziat V, Guerci B, Laville M, Narbonne H, Bodemer C, Lascols O, Capeau J and Vigouroux C: Human lipodystrophies linked to mutations in A-type lamins and to HIV protease inhibitor therapy are both associated with prelamin A accumulation, oxidative stress and premature cellular senescence. Cell Death Differ 14: 1759-1767, 2007.

16. Hennekam RC: Hutchinson-Gilford progeria syndrome: Review of the phenotype. Am J Med Genet A 140: 2603-2624, 2006.

17. Lin F and Worman HJ: Structural organization of the human gene encoding nuclear lamin A and nuclear lamin C. J Biol Chem 268: 16321-16326, 1993.

18. Bergo MO, Gavino B, Ross J, Schmidt WK, Hong C, Kendall LV, Mohr A, Meta M, Genant H, Jiang Y, et al: Zmpste24 deficiency in mice causes spontaneous bone fractures, muscle weakness, and a prelamin A processing defect. Proc Natl Acad Sci USA 99: 13049-13054, 2002.

19. Pendás AM, Zhou Z, Cadiñanos J, Freije JM, Wang J, Hultenby K, Astudillo A, Wernerson A, Rodríguez F, Tryggvason K and López-Otín C: Defective prelamin A processing and muscular and adipocyte alterations in Zmpste24 metalloproteinase-deficient mice. Nat Genet 31: 94-99, 2002.

20. Casey PJ and Seabra MC: Protein prenyltransferases. J Biol Chem 271: 5289-5292, 1996.

21. Maltese WA: Posttranslational modification of proteins by isoprenoids in mammalian cells. FASEB J 4: 3319-3328, 1990.

22. Coffinier C, Hudon SE, Farber EA, Chang SY, Hrycyna CA, Young SG and Fong LG: HIV protease inhibitors block the zinc metalloproteinase ZMPSTE24 and lead to an accumulation of prelamin A in cells. Proc Natl Acad Sci USA 104: 13432-13437, 2007.

23. Young SG, Yang SH, Davies BS, Jung HJ and Fong LG: Targeting protein prenylation in progeria. Sci Transl Med 5: $171 \mathrm{ps} 3,2013$. 
24. Gordon LB, Massaro J, D'Agostino RB Sr, Campbell SE, Brazier J, Brown WT, Kleinman ME and Kieran MW; Progeria Clinical Trials Collaborative: Impact of farnesylation inhibitors on survival in Hutchinson-Gilford progeria syndrome. Circulation 130: 27-34, 2014

25. Lefèvre C, Auclair M, Boccara F, Bastard JP, Capeau J, Vigouroux C and Caron-Debarle M: Premature senescence of vascular cells is induced by HIV protease inhibitors: Implication of prelamin A and reversion by statin. Arterioscler Thromb Vasc Biol 30: 2611-2620, 2010

26. Pistell PJ, Gupta S, Knight AG, Domingue M, Uranga RM, Ingram DK, Kheterpal I, Ruiz C, Keller JN and Bruce-Keller AJ: Metabolic and neurologic consequences of chronic lopinavir/ritonavir administration to $\mathrm{C} 57 \mathrm{BL} / 6$ mice. Antiviral Res 88: 334-342, 2010.

27. ProtM,HeripretL,Cardot-Leccia N,Perrin C,AouadiM,LavrutT, Garraffo R, Dellamonica P, Durant J, Le Marchand-Brustel Y and Binétruy B: Long-term treatment with lopinavir-ritonavir induces a reduction in peripheral adipose depots in mice. Antimicrob Agents Chemother 50: 3998-4004, 2006.

28. Martínez E, Domingo P, Galindo MJ, Milinkovic A, Arroyo JA Baldovi F, Larrousse M, León A, de Lazzari E and Gatell JM: Risk of metabolic abnormalities in patients infected with HIV receiving antiretroviral therapy that contains lopinavir-ritonavir Clin Infect Dis 38: 1017-1023, 2004

29. Montes ML, Pulido F, Barros C, Condes E, Rubio R, Cepeda C, Dronda F, Antela A, Sanz J, Navas E, et al: Lipid disorders in antiretroviral-naive patients treated with lopinavir/ritonavir-based HAART: Frequency, characterization and risk factors. J Antimicrob Chemother 55: 800-804, 2005

30. Saladin R, De Vos P, Guerre-Millo M, Leturque A, Girard J, Staels B and Auwerx J: Transient increase in obese gene expression after food intake or insulin administration. Nature 377 527-529, 1995

31. Maffei M, Halaas J, Ravussin E, Pratley RE, Lee GH, Zhang Y, Fei H, Kim S, Lallone R, Ranganathan S, et al: Leptin levels in human and rodent: Measurement of plasma leptin and ob RNA in obese and weight-reduced subjects. Nat Med 1: 1155-1161, 1995.

32. Yamauchi T, Kamon J, Waki H, Terauchi Y, Kubota N, Hara K, Mori Y, Ide T, Murakami K, Tsuboyama-Kasaoka N, et al: The fat-derived hormone adiponectin reverses insulin resistance associated with both lipoatrophy and obesity. Nat Med 7: 941-946, 2001

33. Tong Q, Sankalé JL, Hadigan CM, Tan G, Rosenberg ES, Kanki PJ, Grinspoon SK and Hotamisligil GS: Regulation of adiponectin in human immunodeficiency virus-infected patients: Relationship to body composition and metabolic indices. J Clin Endocrinol Metab 88: 1559-1564, 2003.

34. Ketlogetswe KS, Post WS, Li X, Palella FJ Jr, Jacobson LP, Margolick JB, Kingsley LA, Witt MD, Dobs AS, Budoff MJ and Brown TT: Lower adiponectin is associated with subclinical cardiovascular disease among HIV-infected men. AIDS 28 901-909, 2014.

35. Das S, Shahmanesh M, Stolinski M, Shojaee-Moradie F, Jefferson W, Jackson NC, Cobbold M, Nightingale P and Umpleby AM: In treatment-naïve and antiretroviral-treated subjects with HIV, reduced plasma adiponectin is associated with a reduced fractional clearance rate of VLDL, IDL and LDL apolipoprotein B-100. Diabetologia 49: 538-542, 2006.
36. Croxtall JD and Perry CM: Lopinavir/Ritonavir: A review of its use in the management of HIV-1 infection. Drugs 70: 1885-1915, 2010.

37. Beck EJ, Vitoria M, Mandalia S, Crowley S, Gilks CF and Souteyrand Y: National adult antiretroviral therapy guidelines in resource-limited countries: Concordance with 2003 WHO guidelines? AIDS 20: 1497-1502, 2006.

38. Carr A, Samaras K, Burton S, Law M, Freund J, Chisholm DJ and Cooper DA: A syndrome of peripheral lipodystrophy, hyperlipidaemia and insulin resistance in patients receiving HIV protease inhibitors. AIDS 12: F51-F58, 1998

39. van Wijk JP and Cabezas MC: Hypertriglyceridemia, metabolic syndrome, and cardiovascular disease in HIV-infected patients: Effects of antiretroviral therapy and adipose tissue distribution. Int J Vasc Med 2012: 201027, 2012.

40. Bonafe SM, Costa DA, Vaz MJ, Senise JF, Pott-Junior H, Machado RH and Castelo A: A randomized controlled trial to assess safety, tolerability, and antepartum viral load with increased lopinavir/ritonavir dosage in pregnancy. AIDS Patient Care STDS 27: 589-595, 2013.

41. Capell BC, Olive M, Erdos MR, Cao K, Faddah DA, Tavarez UL, Conneely KN, Qu X, San H, Ganesh SK, et al: A farnesyltransferase inhibitor prevents both the onset and late progression of cardiovascular disease in a progeria mouse model. Proc Natl Acad Sci USA 105: 15902-15907, 2008.

42. Epling-Burnette PK and Loughran TP Jr: Suppression of farnesyltransferase activity in acute myeloid leukemia and myelodysplastic syndrome: Current understanding and recommended use of tipifarnib. Expert Opin Investig Drugs 19: 689-698, 2010.

43. Milojkovic Kerklaan B, Diéras V, Le Tourneau C, Mergui-Roelvink M, Huitema AD, Rosing H, Beijnen JH, Marreaud S, Govaerts AS, Piccart-Gebhart MJ, et al: Phase I study of lonafarnib (SCH66336) in combination with trastuzumab plus paclitaxel in Her2/neu overexpressing breast cancer: EORTC study 16023. Cancer Chemother Pharmacol 71: 53-62, 2013.

44. Perez-Ruixo JJ, Zannikos P, Ozdemir V, Franc MA, Francke S and Piotrovsky V: Effect of CYP2D6 genetic polymorphism on the population pharmacokinetics of tipifarnib. Cancer Chemother Pharmacol 58: 681-691, 2006

45. Crul M, de Klerk GJ, Swart M, Weiner L, Palmer PA, Bol CJ, Beijnen JH and Schellens JH: Evaluation of the bioequivalence of tablets and capsules containing the novel anticancer agent R115777 (Zarnestra) in patients with advanced solid tumors. Eur J Drug Metab Pharmacokinet 27: 61-65, 2002.

46. Perez-Ruixo JJ, Piotrovskij V, Zhang S, Hayes S, De Porre P and Zannikos P: Population pharmacokinetics of tipifarnib in healthy subjects and adult cancer patients. Br J Clin Pharmacol 62: 81-96, 2006.

This work is licensed under a Creative Commons Attribution-NonCommercial-NoDerivatives 4.0 International (CC BY-NC-ND 4.0) License. 